\title{
Maternal Substrate Utilization Programs the Development of the Metabolic Syndrome in Male Mice Exposed to High Fat In Utero
}

\author{
KIRSTEN HARTIL, PATRICIA M. VUGUIN, MICHAEL KRUSE, ESTHER SCHMUEL, ARIANA FIALLO, CARLOS VARGAS, \\ MATTHEW J. WARNER, JORGE L. DURAND, LINDA A. JELICKS, AND MAUREEN J. CHARRON
}

\begin{abstract}
Departments of Biochemistry [K.H., P.M.V., M.K., A.F., C.V., M.J.W., M.J.C.], Obstetrics and Gynecology and Women's Health [E.S., M.J.C.], and Physiology and Biophysics [J.L.D., L.A.J.], Albert Einstein College of Medicine, Bronx, New York 10461; Department of Pediatrics [P.M.V.], Children's Hospital, Montefiore Medical Center, Bronx, New York 10467
\end{abstract}

\begin{abstract}
Studies were conducted to determine whether maternal substrate utilization during pregnancy affects fetal growth and predisposes offspring to metabolic disease. Female wild-type (WT) and glucose transporter 4 heterozygous mice (G4 \pm , a model of altered peripheral substrate utilization) were fed high-fat diet (HFD, $35.5 \%$ fat) or control chow (C, 9.5\% fat) for 2 wk before mating, throughout pregnancy and lactation (IU/L). WT HFD females exhibited increased serum nonesterified fatty acid and lactate levels and increased hepatic mRNA expression of peroxisome proliferatoractivated receptor $\gamma$ coactivator-1- $\beta$ and SREBP-1c, consistent with increased lipogenesis. G4 \pm HFD females exhibited enhanced lipid clearance, and exposure to HFD did not increase hepatic gene expression. HFD independent of maternal genotype decreased fetal growth and birth weight. WT offspring were weaned onto a low-fat diet (5.6\% fat). Male offspring of WT mothers exposed to HFD exhibited "catch-up" growth accompanied by increased adiposity, impaired glucose tolerance, and insulin sensitivity. In contrast, male offspring of G4 \pm HFD mothers did not exhibit any characteristics of metabolic syndrome. These data suggest that differences in maternal substrate utilization influence offspring metabolic phenotype. (Pediatr Res 66: 368-373, 2009)
\end{abstract}

$\mathrm{T}$ he global epidemic of metabolic syndrome $(1,2)$ cannot be explained entirely by genetics and/or lifestyles. Data suggest that predisposition to these adult diseases has an important portion of their origin in the intrauterine (IU) and early postnatal environment (3). Epidemiologic and animal studies have demonstrated that incidence of type 2 diabetes mellitus (T2DM) and obesity is increased in offspring whose mothers were themselves diabetic or obese before pregnancy (4),

Received January 5, 2009; accepted May 19, 2009.

Correspondence: Maureen J. Charron, PhD, Department of Biochemistry, 1300 Morris Park Avenue, Bronx, NY 10461; e-mail: charron@aecom.yu.edu

Supported by grants from the National Institutes of Health R01DK47425, R01HL73163, R01HL58119, R21 DK081194, P50 DK051296, the Diabetes Research and Training program P60-DK020541, and Cancer Centers of Albert Einstein College of Medicine (M.J.C.) and KO8 HD042172, and an Independent Research Award from Pfizer Inc. (P.V.). K.H. was supported by a Mentor-Based Postdoctoral Fellowship awarded to M.J.C. from the American Diabetes Association.

K.H. and P.M.V. contributed equally to this manuscript.

E. Schmuel is currently at Department of Obstetrics and Gynecology, Harbor-UCLA Medical Center, 1000 West Carson Street, Box 3,Torrance, CA 90502. perpetuating the prevalence of metabolic syndrome and T2DM.

Consumption of Western-style high-fat diets (HFD) increases the risk for gestational diabetes mellitus (GDM), impaired glucose tolerance (5), and preeclampsia during pregnancy (6). In animal models, exposure to HFD IU results in impaired glucose homeostasis, cardiovascular dysfunction, programming of hepatic metabolism, and insulin resistance (7). Little is known about the interaction between consumption of a Western-style HFD during pregnancy and maternal alterations in substrate utilization in programming metabolic disease in offspring. Fetal development is dependent on maternal supply of nutrients, and an altered maternal metabolism would expose the fetus to an unfavorable balance of energy substrate that may predispose offspring to adult disease (8). Mice with homozygous deletion of glucose transporter 4 (GLUT4) alter their substrate utilization to maintain normoglycemia in the absence of GLUT4 (9). Mice with heterozygous deletion of GLUT4 expression (G4 \pm ) have impaired skeletal muscle insulin-stimulated glucose transport and whole body glucose utilization $(10,11)$. In this study, we used female G4 \pm mice as a novel model of altered substrate utilization to determine maternal factors that may contribute to the development of metabolic abnormalities in offspring.

\section{RESEARCH DESIGN AND METHODS}

\footnotetext{
Abbreviations: BW, body weight; C, control breeding chow; CRL, crownrump length; e, embryonic day; G4 $\mathbf{4}$, heterozygous deletion of glucose transporter 4; GLUT4, glucose transporter 4; GTT, glucose tolerance test; HFD, high-fat diet; ITT, insulin tolerance test; IU, In Utero; L, lactation; T2DM, type 2 diabetes mellitus; TG, triglycerides
} 
of a copulatory plug and defined as embryonic day (e) 0.5. Genotyping was performed as previously described (13). Offspring were weaned onto PicoLab Mouse Diet \#5053 (low fat, LF: $5.6 \%$ fat as soybean oil, $20 \%$ protein, $54.8 \%$ carbohydrate $\mathrm{wt} / \mathrm{wt}, 3.4 \mathrm{kcal} / \mathrm{g}$ ) at postnatal d 21 . WT offspring were the focus of this study to eliminate potential confounding effects of genetic factors related to postnatal insulin resistance. Animal protocols were approved by the Animal Institute at the Albert Einstein College of Medicine.

Euglycemic/hyperinsulinemic clamps. Euglycemic clamps were performed in conscious, unrestrained, catheterized adult female mice (27-32 g) as previously described $(11,14)$.

Serum analysis. Fed-state blood samples were collected between $2300 \mathrm{~h}$. and $0100 \mathrm{~h}$. Blood glucose concentrations were measured using a glucometer (Precision Q.I.D., a gift from Abbott Laboratories, Chicago, IL). Commercially available kits were used for measuring serum insulin, adiponectin, (Linco Research, St. Charles, MO), nonesterified fatty acids (NEFAs) (Wako Chemicals, Neuss, Germany), triglycerides (TG), glucose (Sigma Chemical Co., St. Louis, MO), and lactate (Trinity Biotech, St. Louis, MO).

Maternal BW gain during pregnancy. Maternal BW gain during pregnancy was calculated as [(BW day $X-\mathrm{BW}$ e0.5)/BW day mating e18.5] $\times 100$.

Food intake. Mice were housed individually for $4 \mathrm{~d}$ before recording BW and daily food consumption $(\mathrm{kcal} / \mathrm{BW} / \mathrm{d})$ for a period of 5-8 d. Pregnant mice were placed in individual cages on $\mathrm{e} 0.5$, food intake was assessed daily from e 0.5 until e18.5. Food intake in offspring was determined in five mice randomly selected from two to four litters per group.

Maternal organ harvest and fetal data collection. Pregnant mice (WT C, $n=10$; WT HFD, $n=8$; G4 $\pm \mathrm{C}, n=5$; G4 \pm HFD, $n=8$ ) were killed on e18.5; organs were dissected, weighed, and frozen in liquid nitrogen. Litter number, placental and fetal weights, and crown-rump length (CRL) were recorded, as well as the number of abnormal or dead pups per litter. Fetuses were euthanized by cervical dislocation immediately after dissection from the uterine horn.

Oral lipid challenge. Following a 5-h fast, mice received an oral gavage $(16.7 \mathrm{~mL} / \mathrm{kg} \mathrm{BW})$ of olive oil as described previously (9). Serum was collected at the indicated times for TG determination.

Intraperitoneal insulin and GTT. For insulin tolerance tests (ITT), $6 \mathrm{~h}$ fasted mice received an intraperitoneal (i.p.) injection of insulin $(0.75 \mathrm{U} / \mathrm{kg}$ BW) (Humalog, Lilly, Indianapolis, IN). For GTT, overnight fasted mice received an i.p. injection of D-(+)-glucose $(1.5 \mathrm{~g} / \mathrm{kg} \mathrm{BW})$. Blood glucose levels were determined at the indicated times.

Body composition. Body composition (\% body fat) $(n=5 /$ group randomly selected from two to four litters per group, males: $7-8 \mathrm{wk}$, females: $6-7 \mathrm{wk}$ ) was determined using MRI as previously described (15) or by magnetic resonance spectroscopy using an ECHO magnetic resonance spectroscopy instrument (Echo Medical Systems, Houston, TX) (16).

Quantitative real-time PCR analysis. mRNA expression was determined in livers from pregnant mothers as previously described (17). Primers were designed using Primer Express software (Applied Biosystems), and sequences are available on request. Data are expressed relative to the WT C group using the average of the constitutive gene cyclophilin $b$.

Data analysis. Data represents the mean \pm SEM. Statistical analyses were performed using JMP IN 5.1 software (SAS Institute, Cary, NC) or GraphPad Prism software version 5.00 for Windows (GraphPad Software, San Diego California USA, www.graphpad.com). ANOVA was used to test the difference between the means of two ( $t$ test $)$ or more groups. Growth curves, daily food intake, ITT, GTT, and oral lipid clearance experiments were analyzed by two-way repeated-measures ANOVA with Bonferroni posttests.

\section{RESULTS}

Nonpregnant G4 \pm females exhibit decreased insulin sensitivity on a $C$ diet. Euglycemic hyperinsulinemic clamps were performed on 18- to 20-wk-old ( $n=5 /$ group), BW-matched, WT, and G4 \pm mice. Steady-state plasma glucose levels were maintained at similar levels in both genotypes $(119 \pm 0.01$ versus $127 \pm 0.01 \mathrm{mg} / \mathrm{dL}$, WT versus $\mathrm{G} 4 \pm$, respectively, $p=$ NS). Although insulin-mediated suppression of hepatic glucose production during physiologic hyperinsulinemia was similar in both groups $(9.7 \pm 3.1$ versus $6.1 \pm 3.9 \mathrm{mg} / \mathrm{kg} / \mathrm{min}$, WT versus $\mathrm{G} 4 \pm, p=\mathrm{NS}$ ), glucose infusion rate (GIR) required to maintain normoglycemia was $50 \%$ lower in G4 compared with WT mice $(5.7 \pm 0.4$ versus $2.5 \pm 0.4 \mathrm{mg} / \mathrm{kg} / \mathrm{min}$, WT versus $\mathrm{G} 4 \pm$, respectively, $p=0.001$ ). Decreased GIR was largely accounted for by decreased whole body insulin-mediated glucose uptake in $\mathrm{G} 4 \pm$ mice compared with WT mice $(6.6 \pm 0.9$ versus $3.6 \pm 0.6 \mathrm{mg} / \mathrm{kg} / \mathrm{min}$, WT versus $\mathrm{G} 4 \pm$, respectively, $p=$ 0.04 ), demonstrating that $\mathrm{G} 4 \pm$ females exhibit peripheral, but not hepatic, insulin resistance.

Nonpregnant G4 \pm females exhibit increased lipid clearance on HFD. No significant difference in BW was observed in WT or G4 \pm females after 2 wk on a HFD compared with a $\mathrm{C}$ diet (Table 1). WT HFD mice exhibited increased NEFA and free glycerol levels and decreased TG levels compared with WT C (Table 1). HFD did not alter the serum profile in G4 \pm mice, suggesting that G4 \pm is resistant to the acute effect of HFD.

Because of the altered serum lipid profile, the ability to clear orally administrated lipids was assessed (Fig. 1). G4 \pm HFD mice exhibited the lowest serum TG levels, this may be due to an improved ability to clear TG.

HFD did not affect insulin and glucose tolerance (Fig. 1). Despite not having significant differences in the ITT, G4 \pm females were insulin resistant by a clamp study, the gold standard method for measuring insulin action in vivo.

HFD during pregnancy decreases litter weight. Birth weight of offspring exposed to HFD IU was decreased compared with that of offspring exposed to $\mathrm{C}$ diet independent of maternal genotype (Table 2). HFD also decreased the number of pups per litter, although this only reached significance in WT HFD group (Table 2).

Table 1. Effect of acute HFD on metabolic profile

\begin{tabular}{|c|c|c|c|c|}
\hline & WT C $(n=10)$ & $\mathrm{G} 4 \pm \mathrm{C}(\mathrm{n}=8)$ & WT HFD $(n=10)$ & G4 $\pm \operatorname{HFD}(n=8)$ \\
\hline Age (wk) & $13.7 \pm 0.7$ & $15.2 \pm 0.8$ & $14.6 \pm 0.7$ & $15.0 \pm 0.9$ \\
\hline Body weight (g) & $36.8 \pm 1.9$ & $39.1 \pm 2.3$ & $39.0 \pm 2.0$ & $40.2 \pm 2.6$ \\
\hline Food intake $(\mathrm{kcal} / \mathrm{d} / \mathrm{g} \mathrm{BW})$ & $0.48 \pm 0.07$ & $0.46 \pm 0.07$ & $0.38 \pm 0.05$ & $0.34 \pm 0.3$ \\
\hline Glucose $(\mathrm{mg} / \mathrm{dL})$ & $125.8 \pm 2.5$ & $137.0 \pm 4.0$ & $123.5 \pm 6.3$ & $137.4 \pm 6.2$ \\
\hline Insulin $(\mathrm{ng} / \mathrm{mL})$ & $2.27 \pm 1.0$ & $4.64 \pm 1.4$ & $2.91 \pm 1.1$ & $4.21 \pm 1.32$ \\
\hline Lactate (mg/dL) & $47.9 \pm 3.9$ & $38.8 \pm 5.0$ & $53.5 \pm 4.1$ & $40.7 \pm 5.0$ \\
\hline NEFA $(\mu \mathrm{Eq} / \mathrm{mL})$ & $2.36 \pm 0.19$ & $2.43 \pm 0.25$ & $3.19 \pm 0.19 \dagger$ & $2.77 \pm 0.29$ \\
\hline Triglyceride (mg/dL) & $60.45 \pm 6.9$ & $37.57 \pm 8.5^{*}$ & $36.58 \pm 6.9 *$ & $21.93 \pm 8.5^{*}$ \\
\hline Free glycerol (mg/dL) & $110.2 \pm 15.1$ & $121.9 \pm 16.8$ & $144.1 \pm 5.3^{*}$ & $123.2 \pm 6.2$ \\
\hline Adiponectin (ng/mL) & $15.12 \pm 2.1$ & $16.86 \pm 2.5$ & $12.41 \pm 2.5$ & $17.98 \pm 2.8$ \\
\hline
\end{tabular}

Age-matched female mice were placed on HFD or maintained on control diet for 2 wk before the determination of fed metabolic profile. Data are presented as mean $\pm \mathrm{SE}$.

$* p<0.05$ vs WT C.

$\dagger p<0.05$ vs control (C) diet. 

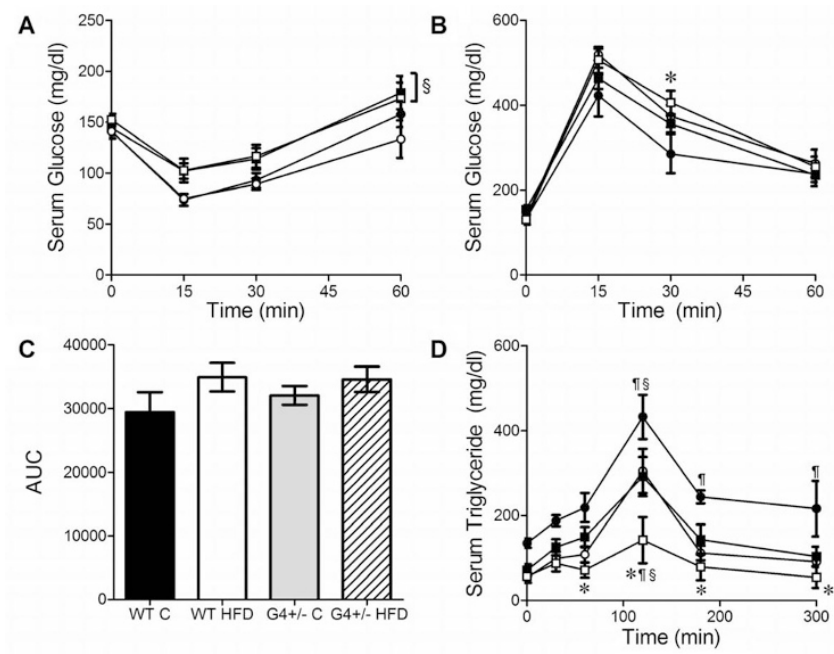

Figure 1. Effect of HFD on $(A)$ ITT, $(B)$ GTT, $(C)$ area under the GTT curve, and $(D)$ lipid clearance in WT and G4 \pm females. WT C (black circles), WT HFD (white circles), G4 $\pm \mathrm{C}$ (black squares), and G4 \pm HFD (white squares) mice. $n=5-7$ per group. ${ }^{*} p<0.05 v s$. WT C, $\$ p<0.05 v s$. WT HFD, $\llbracket p<$ 0.05 vs. G4 \pm C.

HFD alters weight gain during pregnancy. Because of decreased litter weight with HFD, the effects of HFD on maternal BW gain and food intake were assessed. Groups exposed to HFD gained significantly more weight in the first half of pregnancy (e0.5-e9.5) compared with $\mathrm{C}$ groups $(0.74 \pm 0.76,1.23 \pm 0.37$ versus $3.97 \pm 0.80$, and $3.27 \pm$ $0.38 \mathrm{~g}$, WT C, G4 $\pm \mathrm{C}$ versus WT HFD, and G4 \pm HFD, respectively; $p<0.005$ ) (Fig. $2 \mathrm{~A}$ and $\mathrm{B}$ ). In the second half of pregnancy (e10.5-e17.5), HFD groups gained less weight compared with $\mathrm{C}$ groups $(20.02 \pm 2.71,23.82 \pm 1.18$, $14.17 \pm 3.55$, and $13.05 \pm 2.66 \mathrm{~g}$, WT C, G4 $\pm \mathrm{C}$, WT HFD, and G4 \pm HFD, respectively; $p<0.02$ ).

HFD groups consumed more calories from e 0.5 to e9.5 compared with $C$ groups $(110 \pm 9,131 \pm 5,172 \pm 12$, and $151 \pm 10 \mathrm{kcal}$, WT C, G4 \pm C, WT HFD, and G4 \pm HFD, respectively, $p<0.005$ ) (Fig. 2C). No difference in total calories was observed from e10.5 to e17.5 (169 $\pm 7,167 \pm 6$, $171 \pm 13$, and $162 \pm 9 \mathrm{kcal}$, WT C, G4 \pm C, WT HFD, and G4 \pm HFD, respectively; $p=\mathrm{NS}$ ) (Fig. 2C and D). There was no significant difference in total protein consumption during pregnancy among groups $(65 \pm 5,65 \pm 3,55 \pm 4$, and $53 \pm 4 \mathrm{kcal}$, WT C, G4 \pm C, WT HFD, and G4 \pm HFD, respectively, $n=$ 7-12 mice/group; $p=\mathrm{NS}$ ).

HFD decreases fetal size but not litter number or maternal organ weights. To determine how fetal and maternal organ weights contributed to differences in weight gain during pregnancy, mice were killed at e18.5. No difference in number of fetuses per litter was seen $(11.3 \pm 0.9,9.4 \pm 0.9,10.1 \pm 0.8$, and $10.6 \pm 0.8$, WT C, G4 \pm C, WT HFD, and G4 \pm HFD, $n=57-84$ fetuses from five to 10 litters per group) and was present at expected Mendelian frequencies (49 versus 51\%, WT versus G4 \pm fetuses, respectively, $n=306$ fetuses from 31 litters; $p=\mathrm{NS}$ ). Fetal genotype did not affect BW, CRL, or placental weight (data not shown). Exposure to HFD decreased fetal BW $(1.46 \pm 0.02,1.51 \pm 0.02,1.28 \pm 0.02$ II and $1.23 \pm 0.02 * \mathrm{~g}$, WT C, G4 $\pm \mathrm{C}$, WT HFD and G4 \pm HFD, $\mathbb{I} p<0.05$ versus $\mathrm{WT}-\mathrm{C},{ }^{*} p<0.05$ versus $\mathrm{G} 4 \pm \mathrm{C}$ ), and CRL (data not shown), but not placental weight (data not shown), independent of maternal genotype. Maternal BW, liver, heart, and visceral fat pad weights did not differ between groups (data not shown). HFD was associated with an increased liver/ BW ratio in WT compared with G4 \pm mice $(0.036 \pm 0.001$, $0.042 \pm 0.002,0.039 \pm 0.003$, and $0.035 \pm 0.003 \mathrm{~g} / \mathrm{g}$, WT C, G4 \pm C, WT HFD, and G4 \pm HFD, respectively; $p<0.05$ ).

Exposure to HFD IU decreased fetal growth but not litter size, suggesting that decreased pup number in HFD IU litters is due to postnatal mortality (Table 2).

WT HFD mothers exhibit increased expression of genes associated with hepatic lipogenesis. Expression of PPAR $\alpha$ was increased $94 \%$ in WT HFD livers compared with WT C. No differences in the expression of the PPAR $\alpha$ target genes MCAD and LCAD, key enzymes involved in the oxidation of medium- and long-chain fatty acids, was observed (data not shown). Expression of peroxisome proliferator activated receptor $\gamma$ coactivator-1- $\beta$ (PGC1- $\beta$ ) and sterol regulatory element-binding protein 1-c (SREBP-1c), key regulators of hepatic lipogenesis, were increased to 112 and $127 \%$, respectively, in WT HFD compared with WT C livers. Expression of fatty acid-binding protein 1 (L-FABP1), involved in fatty acid uptake and utilization, was increased $63 \%$ in WT HFD livers compared with WT C. No significant alterations in gene expression were seen in G4 \pm livers, suggesting that G4 \pm mice were resistant to the effects of HFD to induce lipogenic gene expression (Fig. 3). Increased lipogenesis in WT HFD livers may explain why only WT HFD developed altered lipid profile.

Offspring $B W$ and composition is determined by diet and maternal genotype. Male mice born to WT HFD IU/L mothers caught-up to $\mathrm{C} I U / \mathrm{L}$, in contrast, male mice from G4 \pm HFD IU/L mothers did not experience catch-up growth (Fig. 4A). HFD IU/L was associated with decreased numbers of pups/litter compared with $\mathrm{C}$ diet at weaning. Similar to birth data, it reached significance in WT HFD IU/L mothers $(11 \pm 1$, $6 \pm 1,11 \pm 1$ and $8 \pm 1$ average number of pups/litter in WT $\mathrm{C}$, WT HFD, G4 $\pm \mathrm{C}$, and G4 \pm HFD IU/L, $p<0.01$ ). No difference in BW and growth rate was found in female offspring, suggesting that catch-up growth in male offspring born

Table 2. Characteristics of newborn pups

\begin{tabular}{lcccc}
\hline & WT C & G4 \pm C & WT HFD & G4 \pm HFD \\
\hline Pups per litter (litters) & $12.2 \pm 0.9(10)$ & $11.6 \pm 1.2(8)$ & $8.6 \pm 0.8 *(10)$ & $9.4 \pm 0.8(8)$ \\
Pup weight (g) (pups) & $1.61 \pm 0.01(164)$ & $1.59 \pm 0.02(87)$ & $1.49 \pm 0.03 *(111)$ & $1.49 \pm 0.03 *(78)$ \\
Total litter weight (g) & $18.95 \pm 1.54$ & $17.30 \pm 1.57$ & $11.47 \pm 1.01 *$ & $12.6 \pm 1.72$ \\
\hline
\end{tabular}

Litter size and pup weight were determined within $24 \mathrm{~h}$ of birth. Data are presented as mean \pm SEM.

$* p<0.05 v s$ control (C) diet. 

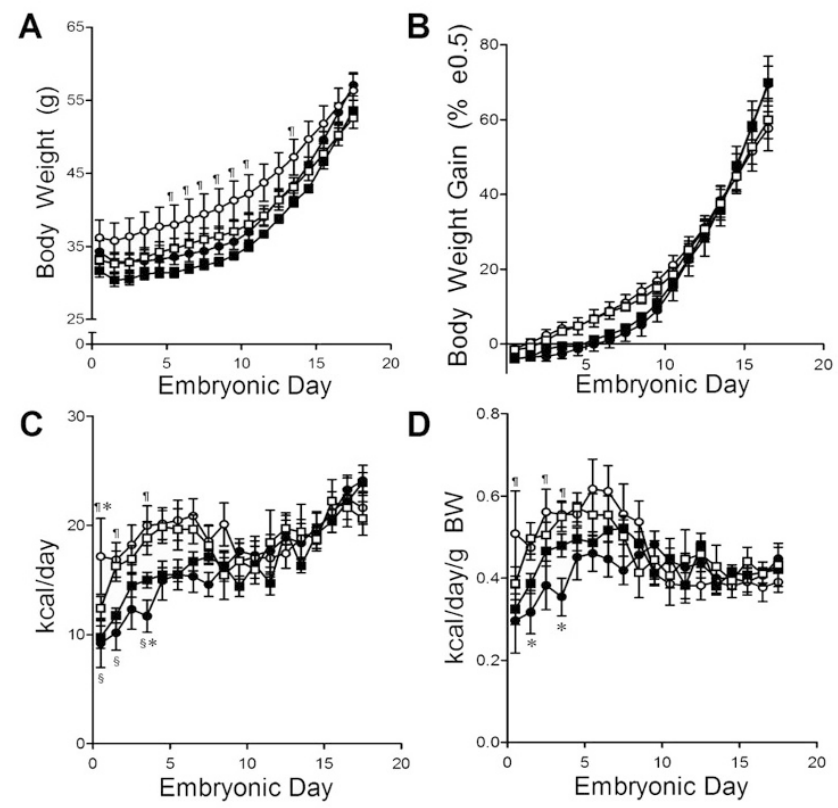

Figure 2. BW gain and food intake during pregnancy. (A) BW and $(B) \%$ BW gain in WT C (black circles), WT HFD (white circles), G4 \pm C (black squares), and G4 \pm HFD (white squares) mice. (C) Daily kilocalories consumed and $(D)$ Daily kilocalories consumed/BW. Four to $10 \mathrm{mice} /$ group. Il $p<0.05$ vs. G4 $\pm \mathrm{C}, \S p<0.05$ vs. WT HFD, $* p<0.05$ vs. G4 \pm HFD.
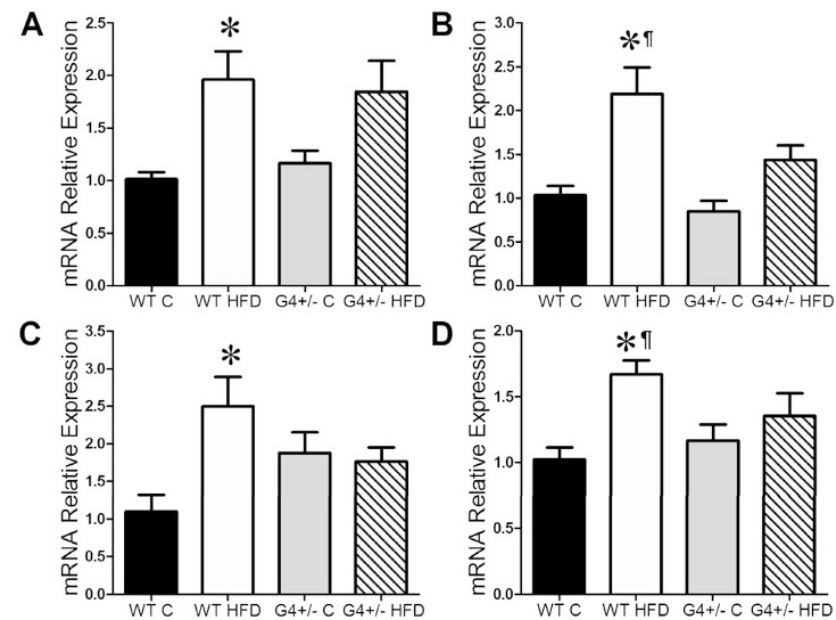

Figure 3. Maternal hepatic gene expression. (A) PPAR $\alpha,(B) \operatorname{PGC} 1 \beta,(C)$ SREBP1-c, and $(D)$ L-FABP1. Five to six mice/group. ${ }^{*} p<0.05 v s$. WT-C, II $v s$. G4 $\pm \mathrm{C}$.

to a WT HFD IU/L mother is not a result of decreased litter size (Fig. 4B).

Adiposity was not different in male offspring of WT and G4 \pm C IU/L mothers $(0.21 \pm 0.02$ versus $0.17 \pm 0.01 \%$, WT C IU/L, G4 \pm C IU/L). In contrast, male offspring from WT HFD IU/L mothers exhibit increased adiposity compared with mice from G4 \pm HFD IU/L mothers $(0.28 \pm 0.01$ versus $0.17 \pm$ $0.01 \%$, WT HFD IU/L versus G4 \pm HFD IU/L, $p<0.0001$ ). Female offspring of G4 \pm HFD IU/L mothers exhibited the greatest adiposity $(0.13 \pm 0.01,0.14 \pm 0.01,0.15 \pm 0.02$, and $0.20 \pm 0.02 \%$, WT C IU/L, G4 \pm C IU/L, WT HFD IU/L, and G4 \pm HFD IU/L, respectively, $p=0.054$,). Thus, in male off-
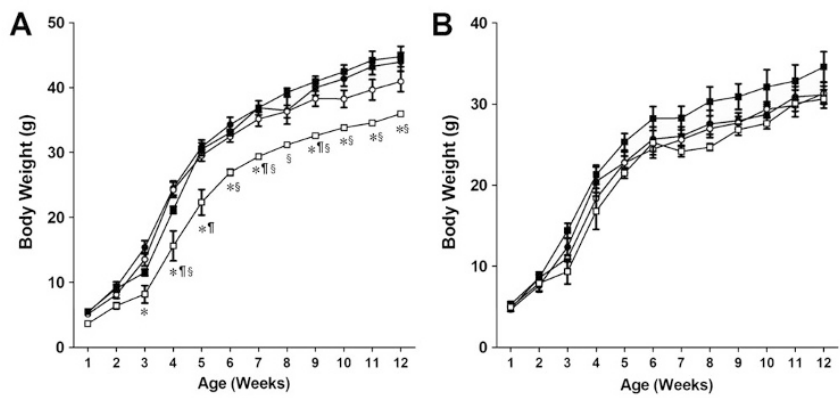

Figure 4. Effect of IU/L diet and maternal genotype on BW of $(A)$ male and $(B)$ female offspring. BW was determined weekly until 12 wk of age. Growth curves of WT offspring of WT C (black circles), WT HFD (white circles), G4 $\pm \mathrm{C}$ (black squares), and G4 \pm HFD (white squares) mothers. $n=4-11$ mice randomly selected from three to eight litters per group, where more than one mouse/litter was represented, the average pup weight/litter was used. $* p<0.05 v s$. WT C, $\S p<0.05 v s$. WT HFD, Ip $<0.05 v s$. G4 \pm C.
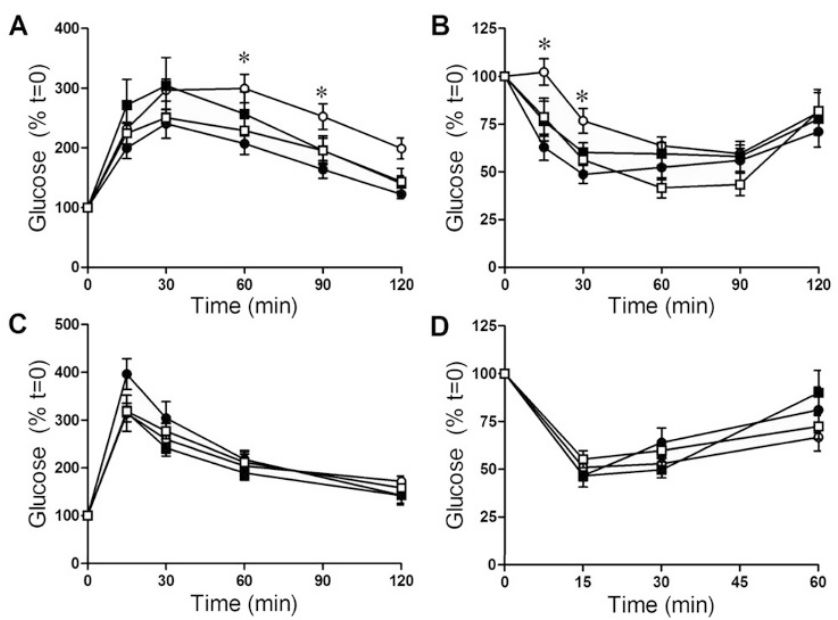

Figure 5. GTT and ITT in $(A$ and $B)$ male and $(C$ and $D)$ female offspring. GTT and ITT were performed on age- and sex-matched 8- to 12-wk-old male and female WT offspring of WT C (black circles), WT HFD (white circles), G4 $\pm \mathrm{C}$ (black squares), and G4 \pm HFD (white squares) mice as described in the Methods section. Data are expressed as a percentage of time $=0, n=5$ mice randomly selected from two to four litters per group, ${ }^{*} p<0.05 v s$. WT C.

spring exposed to HFD IU/L, catch-up growth is accompanied by increased fat accumulation.

Increased fat mass in WT HFD IU/L offspring is not accompanied by increased food intake. Daily food intake in 8- to 9-wk-old male mice was not significantly different between the groups $(0.44 \pm 0.02,0.49 \pm 0.02,0.48 \pm 0.03$, and $0.46 \pm 0.02 \mathrm{kcal} / \mathrm{d} / \mathrm{g} \mathrm{BW}$, WT C IU/L, G4 \pm C IU/L, WT HFD IU/L, and G4 \pm HFD IU/L, respectively). Similar data were obtained in female mice (data not shown). Thus, food intake after the period of catch-up growth is not different between groups.

Increased adiposity was associated with decreased insulin action and glucose disposal. No difference in GTT or ITT was observed in male offspring exposed to C IU/L. In contrast, male offspring of WT HFD IU/L mothers had decreased glucose disposal (Fig. 5A) and insulin sensitivity compared with WT C IU/L (Fig. 5B). Baseline glucose levels were not significantly different between male groups for the GTT $(161 \pm 9,135 \pm 16,142 \pm 6$, and $141 \pm 12 \mathrm{mg} / \mathrm{dL}$, WT C 
IU/L, G4 \pm C IU/L, WT HFD IU/L, and G4 \pm HFD IU/L, respectively) or ITT $(221 \pm 10,189 \pm 16,193 \pm 10$, and $196 \pm 12 \mathrm{mg} / \mathrm{dL}$, WT C IU/L, G4 \pm C IU/L, WT HFD IU/L, and G4 \pm HFD IU/L, respectively). No difference in GTT or ITT was observed in female offspring (Figs. 5C and D, respectively). Baseline glucose levels were not significantly different between female groups for the GTT (77 $\pm 7,91 \pm 3,95 \pm 12$, and $93 \pm 4 \mathrm{mg} / \mathrm{dL}$, WT C IU/L, G4 \pm C IU/L, WT HFD IU/L, and G4 \pm HFD IU/L, respectively) or ITT (144 $\pm 11,145 \pm 6$, $147 \pm 7$, and $151 \pm 8 \mathrm{mg} / \mathrm{dL}$, WT C IU/L, G4 \pm C IU/L, WT HFD IU/L, and G4 \pm HFD IU/L, respectively). Increased adiposity in male offspring of WT HFD IU/L mothers was associated with glucose intolerance and insulin resistance.

\section{DISCUSSION}

These studies were performed to determine the effect of an altered maternal metabolic milieu during pregnancy on programming of offspring metabolic phenotype. G4 \pm female mice are insulin resistant and exhibit enhanced lipid clearance, suggesting that deletion of GLUT4 results in a metabolic remodeling of liver and muscle substrate utilization similar to the GLUT4 knockout model (9). In WT female mice, acute (2 wk) HFD did not cause a significant change in insulin action but did increase NEFA and glycerol levels and decreasing without altering TG levels as previously described (18), suggesting that $2 \mathrm{wk}$ on HFD is adequate to alter serum lipids but not insulin sensitivity. It is possible that the lack of significant effect of HFD on insulin action seen in WT female mice will be secondary to the sample size ( $n=5-7 /$ group). However, it is unlikely because a similar sample size was able to successfully detect significant differences in clamp studies.

In contrast, G4 \pm HFD mice exhibit enhanced lipid clearance contributing to their normal lipid profile. G4 \pm HFD mice exhibit hepatic gene expression similar to G4 $\pm \mathrm{C}$ mice, whereas WT HFD female mice had increased expression of PGC1- $\beta$ and SREBP-1c (19). Differences in hepatic gene expression may explain, in part, the difference in the phenotype between WT and G4 \pm mice in response to HFD.

Decreased fetal weight and birth weight observed with HF exposure may be related to decreased food intake and BW gain during gestation $(20,21)$. In humans, the critical period of maternal weight gain in determining birth weight is the second trimester (22). Decreased fetal weight in mice exposed to diets differing in fatty acid and carbohydrate content has been reported in some studies $(20,23)$ but not others (21). Fetal overgrowth in response to chronic maternal HFD is often accompanied by increased maternal adiposity (24). In our model, acute HFD did not alter maternal BW, allowing us to investigate the effects of altered circulating substrates without the confounding effect of maternal obesity. In agreement with human data, decreased BW gain in the latter part of pregnancy was accompanied by decreased fetal and pup weight.

Fetal growth is regulated by interactions between mother, placenta, and fetus $(25,26)$. Alterations in maternal environment may affect placental structure, decreased uterine blood flow, nutrient transport, epigenetic modification of genes, and increased oxidative stress, triggering a fetal maladaptive re- sponse (27). In addition, fetal growth can be secondary to alterations in glucose transport. In our model, decreased GLUT4 expression is unlikely to be the cause of altered fetal growth because maximal fetal growth is attained during the second and third trimesters (22), although GLUT4 expression has been found in the syncytiotrophoblast during first trimester (28). Furthermore, HFD decreased fetal BW, independent of maternal genotype.

Finally, decreased fetal BW could be secondary to decreased protein intake (29). Protein restriction, as a model of fetal programming, use at least a 50\% reduction in protein intake (29). In our model, total protein consumption during pregnancy was not different among groups, suggesting that protein restriction is not responsible for decreased fetal weight. Low birth weight followed by rapid weight gain during early postnatal life "catch-up growth" has been associated with increased long-term risks for the metabolic syndrome $(30,31)$. Catch-up growth was observed in male offspring of WT, but not in G4 \pm HFD mothers. Catch-up growth in male offspring of WT HFD offspring is unlikely to be the result of decreased litter size. The difference in litter size among the groups was an average of three pups per litter; such a difference has not been shown to cause any metabolic alteration later in life. Litter size has to be substantially reduced to three to four pups per litter to produce metabolic syndrome later in life $(32,33)$.

Because the aim of the current study was to look at HFD exposure during pregnancy and lactation, not at the effect of overfeeding during lactation, we believe that not culling was an appropriate strategy. In addition, male offspring of G4 \pm HFD mice did not exhibit catch-up growth, despite having a similar litter size to WT HFD, suggesting that differences in litter size do not explain the difference in growth rate between offspring from WT and G4 \pm HFD mothers.

Animals that experienced catch-up growth had increased adiposity and exhibited impaired glucose tolerance and insulin sensitivity. Male offspring from G4 \pm HFD IU/L mothers did not exhibit catch-up growth and exhibit normal glucose tolerance and insulin action. Thus, differences in maternal substrate utilization may be the reason why male offspring did or did not develop metabolic abnormalities.

We found a sexually dimorphic response to the same prenatal insult. Sex differences are observed in the pathophysiological response to an adverse fetal environment $(34,35)$. The role of sex hormones and chromosomes, and the regulatory pathways of sexual differentiation, may explain the sexual dimorphism seen in fetal programming. Female mice exposed to HFD IU/L have a normal metabolic response when weaned onto a standard chow diet. However, it is not clear how they will respond to an adverse environment.

We chose a diet relevant to Western societies, where fast food is increasingly popular. In pregnancy, two major dietary patterns are defined: processed meat and high-fat dairy; or vegetables, fruits, poultry and fish. Diets based on processed meat and high fat are associated with fetal growth restriction (36). Two limitations of our study are that it does not discriminate between the specific components of the diet or address the role of the suckling period. Further studies are warranted 
to identify specific macro- or micronutrients as well as the timing of insult that may underlie these associations.

In conclusion, HFD during pregnancy resulted in metabolic syndrome in male WT offspring of WT mothers. In contrast, offspring of G4 \pm HFD mothers seemed to be protected, perhaps because of the altered substrate utilization of G4 \pm HFD mothers. Our novel mouse model offers the potential for further studies into the effects of maternal substrate utilization in response to different diets, on placental function, fetal growth, and metabolic function in the offspring. Future studies will provide additional information on the maternal factors and the critical period in development that determine susceptibility to metabolic syndrome in adulthood.

Acknowledgments. The authors thank Dr. Ellen Katz for many helpful discussions and for assistance in revising this manuscript. We also thank the Diabetes Research and Training Center, Albert Einstein College of Medicine for help with the ECHO MRI and LINCOPLEX analysis.

\section{REFERENCES}

1. Ford ES, Giles WH, Dietz WH 2002 Prevalence of the metabolic syndrome among US adults: findings from the third National Health and Nutrition Examination Survey. JAMA 287:356-359

2. Seidell JC 2000 Obesity, insulin resistance and diabetes-a worldwide epidemic. Br J Nutr 83:S5-S8

3. Hales CN, Barker DJ 2001 The thrifty phenotype hypothesis. Br Med Bull 60:5-20

4. Kral JG 2004 Preventing and treating obesity in girls and young women to curb the epidemic. Obes Res 12:1539-1546

5. Saldana TM, Siega-Riz AM, Adair LS 2004 Effect of macronutrient intake on the development of glucose intolerance during pregnancy. Am J Clin Nutr 79:479-486

6. Scholl TO, Leskiw M, Chen X, Sims M, Stein TP 2005 Oxidative stress, diet, and the etiology of preeclampsia. Am J Clin Nutr 81:1390-1396

7. Taylor PD, Poston L 2007 Developmental programming of obesity in mammals. Exp Physiol 92:287-298

8. Symonds ME, Budge H 2009 Nutritional models of the developmental programming of adult health and disease. Proc Nutr Soc 68:173-178

9. Ranalletta M, Jiang H, Li J, Tsao TS, Stenbit AE, Yokoyama M, Katz EB, Charron MJ 2005 Altered hepatic and muscle substrate utilization provoked by GLUT4 ablation. Diabetes 54:935-943

10. Stenbit AE, Tsao TS, Li J, Burcelin R, Geenen DL, Factor SM, Houseknecht K, Katz EB, Charron MJ 1997 GLUT4 heterozygous knockout mice develop muscle insulin resistance and diabetes. Nat Med 3:1096-1101

11. Rossetti L, Stenbit AE, Chen W, Hu M, Barzilai N, Katz EB, Charron MJ 1997 Peripheral but not hepatic insulin resistance in mice with one disrupted allele of the glucose transporter type 4 (GLUT4) gene. J Clin Invest 100:1831-1839

12. Petry CJ, Ong KK, Dunger DB 2007 Does the fetal genotype affect maternal physiology during pregnancy? Trends Mol Med 13:414-421

13. Katz EB, Stenbit AE, Hatton K, DePinho R, Charron MJ 1995 Cardiac and adipose tissue abnormalities but not diabetes in mice deficient in GLUT4. Nature 377:151155

14. Vuguin P, Raab E, Liu B, Barzilai N, Simmons R 2004 Hepatic insulin resistance precedes the development of diabetes in a model of intrauterine growth retardation. Diabetes 53:2617-2622

15. Capozza F, Combs TP, Cohen AW, Cho YR, Park SY, Schubert W, Williams TM, Brasaemle DL, Jelicks LA, Scherer PE, Kim JK, Lisanti MP 2005 Caveolin-3 knockout mice show increased adiposity and whole body insulin resistance, with ligand-induced insulin receptor instability in skeletal muscle. Am J Physiol Cell Physiol 288:C1317-C1331

16. van de Wall E, Leshan R, Xu AW, Balthasar N, Coppari R, Liu SM, Jo YH, MacKenzie RG, Allison DB, Dun NJ, Elmquist J, Lowell BB, Barsh GS, de Luca C, Myers MG Jr, Schwartz GJ, Chua SC Jr 2008 Collective and individual functions of leptin receptor modulated neurons controlling metabolism and ingestion. Endocrinology 149:1773-1785

17. Ranalletta M, Du XQ, Seki Y, Glenn AS, Kruse M, Fiallo A, Estrada I, Tsao TS, Stenbit AE, Katz EB, Charron MJ 2007 Hepatic response to restoration of GLUT4 in skeletal muscle of GLUT4 null mice. Am J Physiol Endocrinol Metab 293:E1178-E1187

18. Biddinger SB, Almind K, Miyazaki M, Kokkotou E, Ntambi JM, Kahn CR 2005 Effects of diet and genetic background on sterol regulatory element-binding protein1c, stearoyl-CoA desaturase 1, and the development of the metabolic syndrome. Diabetes 54:1314-1323

19. Lin J, Yang R, Tarr PT, Wu PH, Handschin C, Li S, Yang W, Pei L, Uldry M, Tontonoz P, Newgard CB, Spiegelman BM 2005 Hyperlipidemic effects of dietary saturated fats mediated through PGC-1beta coactivation of SREBP. Cell 120:261273

20. Siemelink M, Verhoef A, Dormans JA, Span PN, Piersma AH 2002 Dietary fatty acid composition during pregnancy and lactation in the rat programs growth and glucose metabolism in the offspring. Diabetologia 45:1397-1403

21. Srinivasan M, Katewa SD, Palaniyappan A, Pandya JD, Patel MS 2006 Maternal high-fat diet consumption results in fetal malprogramming predisposing to the onset of metabolic syndrome-like phenotype in adulthood. Am J Physiol Endocrinol Metab 291:E792-E799

22. Sekiya N, Anai T, Matsubara M, Miyazaki F 2007 Maternal weight gain rate in the second trimester are associated with birth weight and length of gestation. Gynecol Obstet Invest 63:45-48

23. Taylor PD, Khan IY, Lakasing L, Dekou V, O'Brien-Coker I, Mallet AI, Hanson MA, Poston L 2003 Uterine artery function in pregnant rats fed a diet supplemented with animal lard. Exp Physiol 88:389-398

24. Jones HN, Woollett LA, Barbour N, Prasad PD, Powell TL, Jansson T 2009 High-fat diet before and during pregnancy causes marked up-regulation of placental nutrient transport and fetal overgrowth in C57/BL6 mice. FASEB J 23:271-278

25. Luther J, Aitken R, Milne J, Matsuzaki M, Reynolds L, Redmer D, Wallace J 2007 Maternal and fetal growth, body composition, endocrinology, and metabolic status in undernourished adolescent sheep. Biol Reprod 77:343-350

26. Remacle C, Bieswal F, Reusens B 2004 Programming of obesity and cardiovascular disease. Int J Obes Relat Metab Disord 28:S46-S53

27. Jansson T, Powell TL 2007 Role of the placenta in fetal programming: underlying mechanisms and potential interventional approaches. Clin Sci (Lond) 113:1-13

28. Ericsson A, Hamark B, Powell TL, Jansson T 2005 Glucose transporter isoform 4 is expressed in the syncytiotrophoblast of first trimester human placenta. Hum Reprod 20:521-530

29. Vuguin PM 2007 Animal models for small for gestational age and fetal programming of adult disease. Horm Res 68:113-123

30. De Blasio MJ, Gatford KL, Robinson JS, Owens JA 2007 Placental restriction of fetal growth reduces size at birth and alters postnatal growth, feeding activity, and adiposity in the young lamb. Am J Physiol Regul Integr Comp Physiol 292:R875R886

31. Crowther NJ, Cameron N, Trusler J, Toman M, Norris SA, Gray IP 2008 Influence of catch-up growth on glucose tolerance and beta-cell function in 7-year-old children: results from the birth to twenty study. Pediatrics 121:e1715-e1722

32. Rodrigues AL, de Moura EG, Passos MC, Dutra SC, Lisboa PC 2009 Postnatal early overnutrition changes the leptin signaling pathway in the hypothalamus-pituitarythyroid axis of young and adult rats. J Physiol 587:2647-2661

33. Schmidt I, Fritz A, Scholch C, Schneider D, Simon E, Plagemann A 2001 The effect of leptin treatment on the development of obesity in overfed suckling Wistar rats. Int J Obes Relat Metab Disord 25:1168-1174

34. Mueller BR, Bale TL 2008 Sex-specific programming of offspring emotionality after stress early in pregnancy. J Neurosci 28:9055-9065

35. Vige A, Gallou-Kabani C, Junien C 2008 Sexual dimorphism in non-Mendelian inheritance. Pediatr Res 63:340-347

36. Knudsen VK, Orozova-Bekkevold IM, Mikkelsen TB, Wolff S, Olsen SF 2008 Major dietary patterns in pregnancy and fetal growth. Eur J Clin Nutr 62:463-470 